\title{
Segundas resecciones anatómicas en pacientes con resección anatómica previa
}

\author{
María Teresa Gómez H. ${ }^{1}$, Nuria Novoa V. ${ }^{1}$, Marta Fuentes G. ${ }^{1}$, \\ Israel Rodríguez A. ${ }^{1}$ y Marcelo F. Jiménez L. ${ }^{1}$
}

'Departamento de Cirugía Torácica. Hospital Universitario de Salamanca, España.

Recibido 2020-05-26 y aceptado 2020-07-09

Correspondencia a: Dra. María Teresa Gómez H. mteresa.gomez.hernandez@ gmail.com

\section{Second pulmonary anatomical lung resections in patients with previous anatomical resections}

Introduction: Second anatomical resections are becoming more frequent in the treatment of synchronous, metachronous and pulmonary metastases of extrapulmonary origin. Aim: The objective of this study is to determine whether second pulmonary anatomical resections are associated with an increased risk of postoperative complications compared to the first intervention. Materials and Method: We have analyzed all patients undergoing a second anatomical resection in our center between October 2000 and February 2019. Complications were classified in major and minor according to the standardized Clavien-Dindo postoperative morbidity classification. The clinical and demographic characteristics of the patients and the occurrence of major complications after the first and second surgical intervention were compared using the $\mathrm{T}$ test for related samples and the McNemar exact test for quantitative and categorical variables, respectively. Results: Seventy-five patients underwent a second anatomic resection. The prevalence of global and major complications after the first intervention was $26.7 \%$ and $4 \%$ compared to $34.7 \%$ and $6.7 \%$ after the second intervention $(p=0.362$ and $p=0.727$, respectively). Second ipsilateral lung resections were associated with $16.7 \%$ of major complications and procedures consisting of completing pneumonectomy with 25\%. Conclusion: Second lung anatomical resections are not associated with an increased risk of postoperative complications compared to the first intervention. However, second ipsilateral resections and resections that involve completing pneumonectomy are associated with a significantly higher risk of major postoperative complications.

Key words: second resection; lobectomy; surgical risk; postoperative complications.

\section{Resumen}

Introducción: Las segundas resecciones anatómicas son cada vez más frecuentes en el tratamiento de carcinomas pulmonares sincrónicos, metacrónicos y de metástasis pulmonares de origen extrapulmonar. Objetivo: Determinar si las segundas resecciones anatómicas pulmonares se asocian con un mayor riesgo de complicaciones posoperatorias comparadas con la primera intervención. Materiales y Método: Hemos analizado todos los pacientes sometidos a una segunda resección anatómica en nuestro centro entre octubre de 2000 y febrero de 2019. Las complicaciones fueron clasificadas en mayores y menores según la clasificación estandarizada de morbilidad posoperatoria de Clavien-Dindo. Se compararon las características clínicas y demográficas de los pacientes y la ocurrencia de complicaciones mayores tras la primera y la segunda intervención quirúrgica mediante la prueba $\mathrm{T}$ para muestras relacionadas y la prueba exacta de McNemar para las variables cuantitativas y categóricas, respectivamente. Resultados: Setenta y cinco pacientes fueron sometidos a una segunda resección anatómica. La prevalencia de complicaciones globales y mayores tras la primera intervención fue del $26,7 \%$ y el $4 \%$ frente al $34,7 \%$ y al $6,7 \%$ tras la segunda intervención ( $\mathrm{p}=0,362$ y $\mathrm{p}=0,727$, respectivamente). Las segundas resecciones pulmonares ipsilaterales se asociaron con un $16,7 \%$ de complicaciones mayores y los procedimientos consistentes en completar la neumonectomía con un 25\%. Conclusión: Las segundas resecciones anatómicas pulmonares no se asocian con un mayor riesgo de complicaciones posoperatorias comparadas con la primera intervención. Sin embargo, las segundas resecciones ipsilaterales y las resecciones que impliquen completar la neumonectomía se asocian con riesgo significativamente superior de complicaciones mayores posoperatorias.

Palabras clave: segunda resección; lobectomía; riesgo quirúrgico; complicaciones posoperatorias. 


\section{Introducción}

El diagnóstico de la neoplasia primaria múltiple pulmonar ha aumentado en los últimos años como consecuencia del incremento de la detección de lesiones pulmonares debido a los avances en las técnicas de imagen ${ }^{1}$, la implementación de programas de cribado de cáncer de pulmón ${ }^{2}$ y al aumento de la supervivencia de los pacientes sometidos a resección pulmonar por un primer cáncer de pulmón ${ }^{3}$. El riesgo de un segundo primario pulmonar en pacientes que han sobrevivido a una resección por cáncer de pulmón no microcítico (CPNM) es aproximadamente de $1 \%-7 \%$ pacientes-año ${ }^{4-6}$ y la resección pulmonar anatómica se considera el tratamiento de elección en estadios iniciales tanto en tumores sincrónicos como metacrónicos ${ }^{7}$. Por otro lado, existe evidencia creciente que apoya el papel de las resecciones repetidas de metástasis pulmonares de origen extrapulmonar ${ }^{8-14}$ en ciertos tipos de cáncer por aportar un beneficio en la supervivencia de pacientes con enfermedad metastásica pulmonar aislada y resecable. Por tanto, las segundas resecciones anatómicas en pacientes con resección anatómica previa son cada vez más frecuentes tanto para el tratamiento de carcinomas pulmonares sincrónicos como metacrónicos y metástasis pulmonares de origen extrapulmonar.

Se estima que la mortalidad y morbilidad cardiorrespiratoria tras resección pulmonar anatómica es del $2,3 \%$ y del $14,9 \%$ respectivamente según el último informe de la Sociedad Europea de Cirujanos Torácicos $(\text { ESTS })^{15}$. Sin embargo, a pesar del reciente incremento del número de segundas resecciones pulmonares en pacientes con resección anatómica previa, se desconoce cuál es el riesgo de complicaciones posoperatorias asociado a estos procedimientos.

El objetivo del estudio es determinar si las segundas resecciones anatómicas pulmonares por cualquier causa se asocian con un mayor riesgo de complicaciones posoperatorias comparadas con la primera intervención.

\section{Materiales y Método}

\section{Población de estudio}

Se incluyeron en el estudio todos los pacientes sometidos de manera consecutiva a una primera y una segunda resección pulmonar anatómica programada (segmentectomía reglada, lobectomía, bilobectomía o neumonectomía) por cualquier causa entre octubre de 2000 y febrero de 2019 en nuestro centro. Fueron excluidos de estudio aquellos pacien- tes sometidos a dos resecciones pulmonares en los que alguna de las dos fuera una resección atípica y aquellos en los que alguna de las dos resecciones se realizara con carácter urgente.

Los criterios de selección de los pacientes fueron uniformes durante todo el período de estudio y se basaron en las guías vigentes ${ }^{16-19}$.

\section{Variables y resultado}

La variable seleccionada como resultado fue la ocurrencia de cualquier complicación posoperatoria y la ocurrencia de complicaciones posoperatorias mayores registradas tras la segunda resección anatómica. Las complicaciones posoperatorias registradas tras la primera resección anatómica sirvieron como control.

Se consideró complicación posoperatoria cualquier evento adverso ocurrido durante el ingreso o en los 30 días tras la intervención: insuficiencia respiratoria (necesidad de ventilación mecánica durante más de $24 \mathrm{~h}$ o necesidad de reintubación en cualquier momento), síndrome de distrés respiratorio agudo, arritmia auricular, arritmia ventricular, atelectasia que requirió broncoscopia, neumonía, tromboembolismo pulmonar, infarto agudo de miocardio, insuficiencia renal, accidente cerebrovascular (ACV), fuga aérea prolongada (definida como la persistencia de fuga aérea a través del drenaje pleural durante más de 5 días tras la intervención quirúrgica), hemotórax, neumotórax con o sin fuga aérea que requiriese drenaje, fístula bronquial, dehiscencia de herida, hematoma de herida, empiema, quilotórax, parálisis recurrencial y parálisis frénica.

Estas complicaciones fueron definidas de antemano de acuerdo con el documento conjunto de definición de variables publicado por las Sociedad Norteamericana (STS) y Europea (ESTS) de Cirujanos Torácicos ${ }^{20} \mathrm{y}$, posteriormente, fueron reclasificadas retrospectivamente según la clasificación estandarizada de morbilidad posoperatoria de Clavien-Dindo ${ }^{21}$ en mayores (grado IIIa a V) y menores (grado I y II).

\section{Análisis estadístico}

Las características clínicas y demográficas de los pacientes registrados en la segunda resección anatómica se compararon con las registradas en la primera resección mediante la prueba $\mathrm{T}$ para muestras relacionadas y la prueba exacta de McNemar para las variables cuantitativas y categóricas, respectivamente.

Con el objeto de determinar si los pacientes eran comparables en cuanto a riesgo en los dos momentos de la cirugía, se calculó la probabilidad de com- 
plicaciones cardiorrespiratorias mediante el modelo de riesgo Eurolung $1^{22}$.

\section{Estimación y comparación de los índices de} complicaciones y complicaciones mayores

Se calculó la prevalencia de cualquier complicación y de complicaciones mayores tras la primera y segunda resección anatómica. Los resultados obtenidos fueron ordenados en tablas 2 × 2 . Para comparar la prevalencia de complicaciones y complicaciones mayores tras la primera y la segunda resección en el mismo paciente, se utilizó la prueba exacta de McNemar para muestras relacionadas.

Igualmente, calculamos la prevalencia de cual-

Tabla 1. Características de la población (variables continuas)

\begin{tabular}{|lllll|}
\hline Variable & & $\mathbf{1}^{\text {a }}$ resección & $\mathbf{2}^{\text {a }}$ resección & P valor \\
Edad & $(\mathrm{M} \pm \mathrm{DT})$ & $63,5 \pm 9,7$ & $65,9 \pm 9,7$ & $\mathrm{p}=0,000$ \\
IMC & $(\mathrm{M} \pm \mathrm{DT})$ & $27,1 \pm 4$ & $27,8 \pm 4$ & $\mathrm{p}=0,001$ \\
VEF1ppo\% & $(\mathrm{M} \pm \mathrm{DT})$ & $73 \pm 17,5$ & $70 \pm 60,1$ & $\mathrm{p}=0,681$ \\
DLCOppo\% & $(\mathrm{M} \pm \mathrm{DT})$ & $71,6 \pm 20,2$ & $62,7 \pm 15,7$ & $\mathrm{p}=0,000$ \\
\hline
\end{tabular}

IMC: índice de masa corporal; VEF1ppo\%: volumen espiratorio forzado en el primer segundo predicho posoperatorio; DLCOppo\%: capacidad de difusión del monóxido de carbono predicha posoperatoria.

Tabla 2. Características de la población (variables dicotómicas)

\begin{tabular}{|lcccl}
\hline Variable & & $\mathbf{1}^{\text {a }}$ resección & $\mathbf{2}^{\text {a }}$ resección & P valor \\
Sexo (hombres) & $\mathrm{n}(\%)$ & $53(70,7 \%)$ & $53(70,7 \%)$ & $\mathrm{p}=1$ \\
EC & $\mathrm{n}(\%)$ & $5(6,7 \%)$ & $5(6,7 \%)$ & $\mathrm{p}=1$ \\
$\mathrm{IRC}$ & $\mathrm{n}(\%)$ & $2(2,7 \%)$ & $2(2,7 \%)$ & $\mathrm{p}=1$ \\
ACV & $\mathrm{n}(\%)$ & $0(0 \%)$ & $0(0 \%)$ & $\mathrm{p}=1$ \\
Resección extendida & $\mathrm{n}(\%)$ & $6(8 \%)$ & $2(2,7 \%)$ & $\mathrm{p}=0,219$ \\
Abordaje VATS & $\mathrm{n}(\%)$ & $13(17,3 \%)$ & $21(28 \%)$ & $\mathrm{p}=0,039$ \\
Neumonectomía & $\mathrm{n}(\%)$ & $0(0 \%)$ & $12(16 \%)$ & $\mathrm{p}=0,000$
\end{tabular}

EC: enfermedad coronaria; IRC: insuficiencia renal crónica; ACV: accidente cerebrovascular; VATS: cirugía toracoscópica video-asistida.

Tabla 3. Diagnóstico anatomopatológico final de la primera y segunda intervención

\begin{tabular}{|lcc|}
\hline Diagnóstico histológico final & $\mathbf{1}^{\mathbf{a}}$ resección & $\mathbf{2}^{\mathbf{a}}$ resección \\
\hline Carcinoma pulmonar & $61(81,3 \%)$ & $52(69,3 \%)$ \\
Metástasis pulmonar & $10(13,3 \%)$ & $13(17,3 \%)$ \\
\hline Nódulo pulmonar sin datos de malignidad & $4(5,3 \%)$ & $10(13,3 \%)$ \\
\hline
\end{tabular}

quier complicación y de complicaciones mayores tras la segunda intervención en función de la lateralidad de la intervención quirúrgica, del procedimiento quirúrgico realizado y del diagnóstico histológico definitivo. Para comparar estas proporciones se utilizó la prueba de chi-cuadrado o el test exacto de Fisher en caso de no cumplirse las condiciones de aplicación de chi-cuadrado.

El análisis estadístico se ha llevado a cabo con el paquete estadístico informático IBM statistics SPSS 22 (IBM Corp, Chicago, Illinois, 2013).

\section{Resultados}

\section{Descripción de la población}

Se analizaron los registros de un total de 75 pacientes sometidos a una segunda resección anatómica. La mediana del intervalo de tiempo entre la primera y la segunda intervención fue de 1,6 años (rango: 21 días-9,9 años). El 68\% de las segundas resecciones fueron contralaterales. El $60 \%$ de las segundas intervenciones consistieron en lobectomías, el 32\% fueron segmentectomías regladas y el $8 \%$ bilobectomías. Globalmente, el 16\% (12 casos) consistieron en completar la neumonectomía.

Las características de la población pueden verse en las Tablas 1 y 2. El diagnóstico anatomopatológico final de la primera y segunda intervención está recogido en la Tabla 3 .

Las probabilidades de complicación cardiorrespiratoria en la primera y segunda resección calculadas mediante el modelo de riesgo Eurolung 1 fueron $0,27( \pm 0,10)$ y $0,29( \pm 0,11),(\mathrm{p}=0,021)$, respectivamente.

\section{Ocurrencia de complicaciones}

La prevalencia de complicaciones tras la primera intervención fue del 26,7\% (20/75); mientras que la proporción de pacientes que presentaron alguna complicación tras la segunda intervención fue del $34,7 \%(26 / 75)$. Estas diferencias no resultaron estadísticamente significativas $(\mathrm{p}=0,362)$. Los resultados pueden verse en la Tabla 4.

En la Tabla 5 se detallan las principales complicaciones posoperatorias presentadas por los pacientes y su prevalencia tras la primera y segunda intervención.

La proporción de pacientes que presentó alguna complicación posoperatoria tras la segunda intervención cuando el procedimiento fue ipsilateral fue el $45,8 \%(11 / 24)$ frente al $29,4 \%(15 / 51)$ en procedimientos contralaterales $(\mathrm{p}=0,163)$. La complicación más frecuente en los procedimientos ipsilaterales fue 
el hemotórax $(16,7 \%)$, mientras que la más frecuente tras resecciones contralaterales fue la fuga aérea prolongada $(11,8 \%)$.

La prevalencia de complicaciones tras completar la neumonectomía fue de 58,3\% (7/12), mientras que en el resto de los pacientes que fueron sometidos a resecciones anatómicas que no implicaron completar la neumonectomía la prevalencia fue del $30,2 \%(19 / 63), p=0,096$.

El 36,5\% (19/52) de los pacientes con diagnóstico de carcinoma de pulmón presentó alguna complicación posoperatoria frente al 30,4\% cuando el diagnóstico definitivo fue metástasis pulmonar o nódulo sin datos de malignidad, $\mathrm{p}=0,609$.

\section{Ocurrencia de complicaciones mayores}

La prevalencia de complicaciones mayores tras la primera intervención fue del 4\% (3/75); mientras que la proporción de pacientes que presentaron alguna complicación tras la segunda intervención fue del $6,7 \%(5 / 75)$; en un caso esta complicación derivó en el fallecimiento del paciente $(1,3 \%)$ al que se le había sido completado la neumonectomía. Estas diferencias no resultaron significativas $(p=0,727)$.

Los resultados pueden verse en la Tabla 6.

La proporción de pacientes que presentó alguna complicación posoperatoria mayor tras la segunda intervención cuando el procedimiento fue ipsilateral fue el $16,7 \%(4 / 24)$ frente al $2 \%(1 / 51)$ en procedimientos contralaterales $(p=0,034)$.

La prevalencia de complicaciones mayores tras completar la neumonectomía fue del 25\% (3/12), mientras que en el resto de pacientes que fueron sometidos a resecciones anatómicas que no implicaron completar la neumonectomía la prevalencia fue del $0,03 \%(2 / 63), \mathrm{p}=0,027$.

El 9,6\% de los pacientes con diagnóstico de carcinoma de pulmón presentó alguna complicación posoperatoria mayor, mientras que no se dio ninguna complicación de este tipo en resecciones por lesiones no malignas o metástasis pulmonares $(\mathrm{p}=0,315)$.

\section{Discusión}

Las segundas resecciones pulmonares anatómicas en pacientes con antecedentes de resección anatómica previa plantean ciertas dificultades en cuanto al manejo perioperatorio del paciente. Así, en el caso de resecciones contralaterales, puede ser complicado mantener una oxigenación adecuada y estable durante la ventilación unipulmonar ${ }^{23}$. Mientras que, en el caso de las resecciones anatómicas ipsilaterales,
Tabla 4. Ocurrencia de complicaciones tras la primera y segunda intervención

\begin{tabular}{|c|c|c|c|}
\hline \multirow{4}{*}{ 1a resección } & \multirow[b]{3}{*}{ Sí complicaciones } & \multicolumn{2}{|c|}{$2^{\mathrm{a}}$ resección } \\
\hline & & Sí complicaciones & No complicaciones \\
\hline & & 8 & 12 \\
\hline & No complicaciones & 18 & 37 \\
\hline
\end{tabular}

Tabla 5. Principales complicaciones tras la primera y la segunda intervención

\begin{tabular}{|lccc|}
\hline Complicación & $\mathbf{1}^{\text {a }}$ resección & $\mathbf{2}^{\text {a }}$ resección & p valor \\
Arritmia & $0(0 \%)$ & $4(5,3 \%)$ & $\mathrm{p}=0,125$ \\
Neumonía & $2(2,7 \%)$ & $4(5,3 \%)$ & $\mathrm{p}=0,688$ \\
Atelectasia & $2(2,7 \%)$ & $1(1,3 \%)$ & $\mathrm{p}=1$ \\
Insuficiencia respiratoria & $1(1,3 \%)$ & $4(5,3 \%)$ & $\mathrm{p}=0,375$ \\
Fuga aérea prolongada & $6(8 \%)$ & $8(10,7 \%)$ & $\mathrm{p}=0,774$ \\
Hemotórax & $3(4 \%)$ & $5(10,7 \%)$ & $\mathrm{p}=0,727$ \\
Reintervención & $1(1,3 \%)$ & $3(4 \%)$ & $\mathrm{p}=0,625$ \\
\hline
\end{tabular}

Tabla 6. Ocurrencia de complicaciones mayores tras la primera y segunda intervención

\begin{tabular}{|c|c|c|c|}
\hline \multirow{4}{*}{$1^{\text {a }}$ resección } & & \multicolumn{2}{|c|}{$2^{a}$ resección } \\
\hline & & $\begin{array}{c}\text { Sí complicaciones } \\
\text { mayores }\end{array}$ & $\begin{array}{c}\text { No complicaciones } \\
\text { mayores }\end{array}$ \\
\hline & $\begin{array}{l}\text { Sí complicaciones } \\
\text { mayores }\end{array}$ & 0 & 3 \\
\hline & $\begin{array}{l}\text { No complicaciones } \\
\text { mayores }\end{array}$ & 5 & 67 \\
\hline
\end{tabular}

éstas pueden complicarse por la presencia de adherencias intensas especialmente aquellas que afectan a las estructuras hiliares ${ }^{24}$. Ambas circunstancias pueden conducir a un incremento del riesgo de complicaciones posoperatorias y mortalidad.

\section{Interpretación de los principales resultados}

Los principales resultados del estudio muestran que, aunque las diferencias detectadas no son estadísticamente significativas, las segundas resecciones tienen un mayor riesgo de complicaciones (globales y mayores) comparadas con la primera.

En el análisis realizado en función de la lateralidad, cabe destacar que las segundas resecciones ipsilaterales se asociaron con una alta prevalencia de complicaciones globales $(45,8 \%$ frente al $29,4 \%$ en procedimientos contralaterales) y mayores (16,7\% frente al $2 \%$ en resecciones contralaterales). Estas 
diferencias que pueden justificarse por la presencia de adherencias, son clínicamente relevantes y deben tenerse en cuenta a la hora de valorar a los pacientes candidatos a segundas resecciones ipsilaterales y en manejo perioperatorio de los mismos.

Como otro hallazgo destacable figura el hecho de que las segundas cirugías consistentes en completar la neumonectomía se asociaron con un riesgo muy alto de complicaciones globales $(58,3 \%)$, mayores (25\%) y una mortalidad del $8,3 \%(1 / 12)$. La presente serie describe un total de 12 casos en los que la segunda intervención consistió en completar la neumonectomía; este procedimiento se asocia con altos índices de complicaciones y mortalidad posoperatorias según estudios previos ${ }^{36,37}$ que podrían haber influido en los resultados asociados a la segunda resección. Sin embargo, hemos considerado oportuno incluirlos en el análisis, puesto que reflejan la práctica clínica real; completar la neumonectomía es un procedimiento frecuente cuando se trata de segundas resecciones ipsilaterales que en nuestra serie alcanzó el 16\% de todas las segundas resecciones y el $50 \%$ de las segundas resecciones ipsilaterales.

Los resultados obtenidos son consistentes con los publicados por otros autores. Así, Shah et al. ${ }^{28}$, publicaron los resultados de 47 pacientes sometidos a resección pulmonar por tumores primarios sincrónicos bilaterales. El 28\% de los pacientes de la serie presentaron complicaciones tras alguna de las dos cirugías, aunque la mayoría fueron menores y sólo el $6 \%$ se consideraron mayores. Además, registraron una muerte perioperatoria ( $2 \%$ ). Igualmente, Finley et al. ${ }^{29}$ y Trousse et al. ${ }^{30}$, analizaron los resultados a corto y largo plazo de pacientes sometidos a resección pulmonar por lesiones primarias sincrónicas tanto unilaterales como bilaterales (175 y 125 casos, respectivamente) y publicaron una morbilidad posoperatoria del $33 \%$ y del $27,2 \%$, respectivamente y una mortalidad a 30 días del 1,2\% y del 4,5\%, respectivamente.

En cuanto a las resecciones quirúrgicas por segundos tumores primarios de pulmón, Battafarano et al. ${ }^{31}$, publicaron los resultados de la resección quirúrgica en cáncer de pulmón metacrónico después de revisar 69 pacientes; la mortalidad posoperatoria registrada e la serie fue del 5,8\% tras la segunda resección. Doddoli et al. ${ }^{32}$, revisaron 38 pacientes intervenidos por cáncer de pulmón metacrónico y describieron una mortalidad posoperatoria del 13\%. La mortalidad posoperatoria tras completar la neumonectomía fue del $7 \%$.

Sin embargo, estos estudios incluyeron pacientes sometidos a resecciones sublobares no anatómicas que se asocian con un menor riesgo de complicacio- nes posoperatorias que las resecciones anatómicas ${ }^{33}$, por lo que consideramos que nuestro estudio aporta información original y relevante al incluir únicamente resecciones anatómicas tanto en la primera como en la segunda intervención.

Igualmente, según los datos publicados en la literatura ${ }^{12,34}$, las resecciones pulmonares repetidas por metástasis de origen extrapulmonar son procedimientos seguros que asocian escasa morbilidad y nula mortalidad posoperatoria, si bien es cierto que las segmentectomías atípicas son las resecciones más frecuentes tanto en la primera como en la segunda intervención.

Por otro lado, Yasuda et al. ${ }^{23}$, publicaron una serie de 14 pacientes con antecedentes de lobectomía previa que se sometieron a una segunda lobectomía contralateral videotoracoscópica sin presentar mortalidad ni complicaciones mayores. De forma similar, Ordoñez et al..$^{35}$, describieron una serie de 6 pacientes sometidos a segundas lobectomía pulmonar en la que excluyeron las segundas lobectomías que implicaron completar la neumonectomía y registraron una prevalencia de complicaciones mayores tras la segunda cirugía del 33\% y una nula mortalidad posoperatoria, resultados similares a los encontrados en nuestra serie. Nuestro estudio incluye un mayor número de pacientes, así como resecciones pulmonares sublobares anatómicas y neumonectomías de compleción.

El presente estudio fue diseñado de manera que cada individuo sirviera como su propio control con objeto de evitar los sesgos inherentes a la varianza natural entre individuos, sin embargo, como es lógico, las características clínicas de los pacientes se modificaron en el período de tiempo transcurrido entre la primera y segunda resección, lo que se tradujo en el incremento estadísticamente significativo del riesgo de complicaciones cardiorrespiratorias asociado a la segunda intervención de acuerdo con el modelo Eurolung 1. Sin embargo, este incremento del riesgo no se vio reflejado en un incremento estadísticamente significativo de la ocurrencia de complicaciones asociadas a la segunda resección.

\section{Limitaciones}

En primer lugar, el protocolo de fisioterapia posoperatoria seguido por los pacientes no fue uniforme durante todo el período de estudio. Sin embargo, sólo fueron 4 los pacientes que no siguieron el protocolo actual por haber sido intervenidos antes de su implementación en noviembre de 2002. Además, ningún caso recibió un manejo posoperatorio diferente en una y otra intervención.

En segundo lugar, no se ha considerado el trata- 
miento adyuvante con quimio o radioterapia tras la primera intervención como posible factor relacionado con la ocurrencia de complicaciones posoperatorias tras la segunda intervención, circunstancia que podría ser especialmente relevante en el caso de las segundas resecciones ipsilaterales de tumores metacrónicos.

Además, este estudio puede tener sesgos inherentes a cualquier análisis retrospectivo. Aunque las complicaciones estaban bien definidas y las variables estandarizadas, no podemos descartar que pueda haber algún error de introducción de los datos, clasificación errónea o notificación insuficiente.

\section{Conclusiones}

Las segundas resecciones anatómicas pulmonares no se asocian con un mayor riesgo de complica- ciones posoperatorias comparadas con la primera intervención. Sin embargo, las segundas resecciones ipsilaterales y las resecciones que impliquen completar la neumonectomía se asocian con riesgo significativamente superior de complicaciones mayores posoperatorias.

\section{Responsabilidades éticas}

Protección de personas y animales. Los autores declaran que para esta investigación no se han realizado experimentos en seres humanos ni en animales.

Confidencialidad de los datos. Los autores declaran que en este artículo no aparecen datos de pacientes.

Conflictos de interés: no hay.

\section{Bibliografía}

1. Shah AA, Barfield ME, Kelsey CR, Onaitis MW, Tong B, Harpole D, et al. Outcomes after surgical management of synchronous bilateral primary lung cancers. Ann Thorac Surg. 2012;93:105560 .

2. Vázquez $\mathrm{M}$, Carter D, Brambilla $\mathrm{E}$, Gazdar A, Noguchi M, Travis WD, et al. Solitary and multiple resected adenocarcinomas after $\mathrm{CT}$ screening for lung cancer: histopathologic features and their prognostic implications. Lung Cancer 2009;64:148-54.

3. Johnson BE, Cortázar P, Chute JP. Second lung cancers in patients successfully treated for lung cancer. Semin Oncol. 1997;24:492-9.

4. Ripley RT, McMillan RR, Sima CS, Hasan SM, Ahmad U, Lou F, et al. Second primary lung cancers: smokers versus nonsmokers after resection of stage I lung adenocarcinoma. Ann Thorac Surg. 2014;98:968-74.

5. Lou F, Huang J, Sima CS, Dycoco J, Rusch V, Bach PB. Patterns of recurrence and second primary lung cancer in early stage lung cancer survivors followed with routine computed tomography surveillance. J Thorac Cardiovasc Surg. 2013;145:75-81.

6. Johnson BE. Second lung cancers in patients after treatment for an initial lung cancer. J Natl Cancer Inst. 1998;90:133545 .

7. NCCN clinical practice guidelines in oncology. Non-small cell lung cancer. Version 6. 2019. Disponible en: https:// www.nccn.org/professionals/physician gls/pdf/nscl.pdf (Último acceso: 22 de septiembre de 2019).

8. Sponholz S, Schirren M, Baldes N, Oguzhan S, Schirren J. Repeat resection for recurrent pulmonary metastasis of colorectal cancer. Langenbecks Arch Surg. 2017;402:77-85.

9. Chudgar NP, Brennan MF, Tan KS, Munhoz RR, D'Angelo SP, Bains MS, et al. Is repeat pulmonary metastasectomy indicated for soft tissue sarcoma? Ann Thorac Surg. 2017;104:1837-45.

10. Kim AW, Faber LP, Warren WH, Saclarides TJ, Carhill AA, Basu S, et al. Repeat pulmonary resection for metachronous colorectal carcinoma is beneficial. Surgery 2008;144:712-7.

11. Chen F, Sakai H, Miyahara R, Bando T, Okubo K, Date H. Repeat resection of pulmonary metastasis is beneficial for patients with colorectal carcinoma. World J Surg. 2010;34:2373-8.

12. Hishida T, Tsuboi M, Okumura T, Boku N, Ohde Y, Sakao Y, et al. Does repeated lung resection provide long-term survival for recurrent pulmonary metastases of colorectal cancer? Results of a retrospective Japanese multicenter study. Ann Thorac Surg. 2017;103:399-405.

13. Menna C, Berardi G, Tierno SM, Andreetti C, Maurizi G, Ciccone AM, et al. Do repeated operations for recurrent colorectal lung metastases result in improved survival? Ann Thorac Surg. 2018;106:421-7.

14. Chen F, Miyahara R, Bando T, Okubo K, Watanabe K, Nakayama T, et al. Repeat resection of pulmonary metastasis is beneficial for patients with osteosarcoma of the extremities. Interact Cardiovasc Thorac Surg. 2009;9:649-53.

15. ESTS Database annual report 2019: http:// www.ests.org/_userfiles/pages/files/ database_reports/ESTS $\% 202018 \% 20$ Silver\%20Book.pdf (Último acceso: 22 de septiembre de 2019).

16. Brunelli A, Kim AW, Berger KI, Addrizzo-Harris DJ. Physiologic evaluation of the patient with lung cancer being considered for resectional surgery: Diagnosis and management of lung cancer, 3rd ed: American College of Chest Physicians evidence-based clinical practice guidelines. Chest 2013;143(5 Suppl):e166S-e190S. Erratum in: Chest. 2014;145:437.

17. Colice GL, Shafazand S, Griffin JP, Keenan R, Bolliger CT. American College of Chest Physicians. Physiologic 
evaluation of the patient with lung cancer being considered for resectional surgery: ACCP evidenced-based clinical practice guidelines (2nd edition). Chest 2007;132(3 Suppl):161S-77S.

18. Beckles MA, Spiro SG, Colice GL, Rudd RM. American College of Chest Physicians. The physiologic evaluation of patients with lung cancer being considered for resectional surgery. Chest 2003;123(1 Suppl):105S-114S.

19. Reilly JJ Jr. Evidence-based preoperative evaluation of candidates for thoracotomy. Chest 1999;116(6 Suppl):474S-476S.

20. Fernández FG, Falcoz PE, Kozower BD, Salati M, Wright CD, Brunelli A. The Society of Thoracic Surgeons and the European Society of Thoracic Surgeons general thoracic surgery databases: joint standardization of variable definitions and terminology. Ann Thorac Surg. 2015;99:368-76.

21. Dindo D, Demartines N, Clavien PA. Classification of surgical complications. A new proposal with evaluation in a cohort of 6336 patients and results of a survey. Annals of Surgery 2004;240:205-13

22. Brunelli A, Salati M, Rocco G, Varela G, Van Raemdonck D, Decaluwe H, et al. European risk models for morbidity (EuroLung1) and mortality (EuroLung2) to predict outcome following anatomic lung resections: an analysis from the European Society of Thoracic Surgeons database. Eur J Cardiothorac Surg. 2017;51:490-7.

23. Yasuda M, Nakanishi R, Shinohara S,
Mori M, Ashikari S, Oyama T, et al. Contralateral thoracoscopic lobectomy in postlobectomy patients. J Thorac Dis. 2017;9:3187-92.

24. Takahashi Y, Miyajima M, Tada M, Maki R, Mishina T, Watanabe A. Outcomes of completion lobectomy long after segmentectomy. J Cardiothorac Surg. 2019;14:116.

25. Yang H, Sun Y, Yao F, Yu K, Gu H, Han B, et al. Surgical Therapy for Bilateral Multiple Primary Lung Cancer. Ann Thorac Surg. 2016;101:1145-52.

26. Rahn DA 3rd, Thakur S, Makani S, Sandhu A. Stereotactic body radiation therapy (SBRT) for multiple primary lung cancers (MPLC): a review and case series. J Radiosurg SBRT 2013;2:135-40.

27. Ginsberg RJ, Rubinstein LV. Randomized trial of lobectomy versus limited resection for T1N0 non-small cell lung cancer. Lung Cancer Study Group Ann Thorac Surg. 1995;60:615-23.

28. Shah AA, Barfield ME, Kelsey CR, Onatis MW, Tong B, Harpole D, et al. Outcomes after surgical management of synchronous bilateral primary lung cancers. Ann Thorac Surg. 2012;93:1055-60.

29. Finley DJ, Yoshizawa A, Travis W, Zhou Q, Seshan VE, Bains MS, et al. Predictors of outcomes after surgical treatment of synchronous primary lung cancers. J Thorac Oncol. 2010;5:197-205.

30. Trousse D, Barlesi F, Loundou A, Tasei AM, Doddoli G, Giudicelli R, et al. Synchronous multiple primary lung cancer: an increasing clinical occurrence requiring multidisciplinary management. J Thorac Cardiovasc Surg. 2007;133:1193200.

31. Battafarano RJ, Force SD, Meyers BF, Bell J, Guthrie TJ, Cooper JD, et al. Benefits of resection for metachronous lung cancer. J Thorac Cardiovasc Surg. 2004;127:836-42.

32. Doddoli C, Thomas P, Ghez O, Giudicelli R, Fuentes P. Surgical management of metachronous bronchial carcinoma. Eur J Cardiothorac Surg. 2001;19:899-903.

33. Sihoe AD, Van Schil P. Non-small cell lung cancer: when to offer sublobar resection. Lung Cancer 2014;86:115-20.

34. Hachimaru A, Maeda R, Suda T, Takagi Y. Repeat pulmonary resection for recurrent lung metastases from colorectal cancer: an analysis of prognostic factors. Interact Cardiovasc Thorac Surg. 2016;22:826-30.

35. Ordóñez Lozano PA, Royo Crespo I, Muñoz-González N, Embún Flor R. Second lung lobectomies: Are they safe and feasible? Arch Bronconeumol. 2018;54:227-8.

36. Puri V, Tran A, Bell JM, Crabtree TD, Kreisel D, Krupnick AS, et al. Completion pneumonectomy: outcomes for benign and malignant indications. Ann Thorac Surg. 2013;95:1885-90.

37. Guggino G, Doddoli C, Barlesi F, Acri P, Chetaille B, Thomas P, et al. Completion pneumonectomy in cancer patients: experience with 55 cases. Eur J Cardiothorac Surg. Eur J Cardiothorac Surg. 2004;25:449-55. 